\title{
El uso eficiente de la energía eléctrica en los ingenios azucareros como contribución al desarrollo sostenible de Nicaragua
}

\author{
The efficient use of electricity in sugar mills as a \\ contribution to sustainable development in Nicaragua
}

Napoleón Vicente Blanco-Orozco'

Eduardo Arce-Díaz ${ }^{2}$

Fecha de recepción: 2 de octubre del 2012

Fecha de aprobación: 4 de diciembre del 2012

Blanco-Orozco, N; Arce-Díaz, E.

Entre la teoría y la práctica de la conservación de los recursos naturales:

las parcialidades I de Totonicapán,

Guatemala. Tecnología en Marcha. Vol.

26, № 3. Pág 84-93

Ingeniero eléctrico. Máster en Gerencia de Proyectos de Desarrollo, 2007. Estudiante de Doctorado en Ciencias Naturales para el Desarrollo en el Instituto Tecnológico de Costa Rica. Docente de la Universidad Nacional de Ingeniería, Facultad de Electrotecnia y Computación, Nicaragua. Correo electrónico: blanconapoleon@yahoo.com, napoleon.blanco@usr.uni.edu.ni; www.docentes.uni.edu.ni/Napoleon.Blanco.

2 Ingeniero Mecánico. M.Sc. Kansas State University, E.U.A. Ingeniería Agrícola, 1988. Ph.D. Kansas State University, E.U.A. Economía Agrícola, 1995. Consultor, instructor y profesor internacional para organizaciones. Costa Rica. Correo electrónico: Eduardo.Arce@ AsesoresEyP.com 


\section{Palabras clave}

Eficiencia; ahorro; energía eléctrica; consumo; educación ambiental

\section{Resumen}

En este artículo se aborda cómo el uso eficiente de la energía eléctrica contribuiría al desarrollo sostenible de Nicaragua y, por ende, a un cambio en la cultura de su utilización por parte del sector industrial, específicamente en los ingenios azucareros. En la actualidad, en Nicaragua se usan principalmente combustibles fósiles para generar energía eléctrica, lo cual causa contaminación ambiental, así como dependencia económica y tecnológica. Esto evidencia la necesidad de gestar un cambio de cultura que implique una forma de consumo eficiente y racional de la energía eléctrica. Para mejorar la eficiencia en el consumo de energía eléctrica y disminuir la alta intensidad energética, se requiere una gestión integral de energía que permita su ahorro. Además, se propone el empleo de tecnologías alternativas, tales como la cogeneración, la energía solar pasiva - la arquitectura bioclimática, domótica y la energía solar térmica de baja temperatura. Por lo tanto, se plantea el cambio de la cultura del uso de la energía, con el fin de emplearla eficientemente $y$, además, aprovechar otras formas alternativas de energía. Finalmente, se considera necesario educar con nuevos valores a los consumidores para construir una nueva cultura energética y ambiental que permita el aumento de la productividad, la competitividad y la reducción de impactos ambientales negativos derivados de la generación de energía eléctrica usando combustibles fósiles.

\section{Key words}

Efficiency; saving; electrical energy; consumption, environmental education

\begin{abstract}
This article addresses how the efficient use of electricity and, thus, changing the culture of its use in the industrial sector of sugar mills, contributes to sustainable development in Nicaragua. It is shown that today, in order to meet the demand of electricity in Nicaragua, fossil fuels are mainly used to generate electricity, which causes environmental pollution and economic and technological dependence. Therefore, it is necessary to promote a change in the culture that involves a form of efficient and rational consumption of electricity. To improve the efficiency in the consumption of electricity of sugar mills and to reduce high energy intensity, it is necessary a comprehensive energy management that allows energy saving. In addition, we propose the use of alternative technologies such as passive solar energy or bioclimatic architecture, automation, cogeneration and low temperature solar thermal energy as ways to reduce the environmental impact caused by the electric power generation using fossil fuels. Therefore, it is proposed to change the culture of energy use in order to employ it efficiently and also take advantage of other alternative forms of energy. Finally, it is necessary to educate consumers in new values to build a new energy and environment culture, allowing to increase productivity, competitiveness, and reduce negative environmental impacts of the generation of the the power generation using fossil fuels.
\end{abstract}




\section{Introducción}

Una manera de contribuir al desarrollo sostenible de Nicaragua es promover el cambio cultural en el uso de la energía eléctrica de la sociedad y de esta forma contribuir a la conservación del medio ambiente, disminuyendo la necesidad de generación de energía eléctrica, dado que en este proceso se emplean primordialmente combustibles fósiles, provocando la emisión de contaminantes. En el mismo sentido, Nicaragua emplea la energía de manera ineficiente en los procesos de producción, es decir, presenta alta intensidad energética. Como alternativa de solución, se propone la eficiencia energética que está relacionada con la cantidad de productos que se obtienen de un proceso por unidad de energía y se define como el conjunto de actividades encaminadas a optimizar el consumo de energía en términos unitarios, manteniendo el nivel de los servicios prestados (Ramírez, 2008). Además, la eficiencia energética está en función de conductas individuales y del nivel de racionalidad en las decisiones de consumo humano de energía, por eso se puede aprender a usar de manera eficiente y de esta forma aportar al desarrollo sostenible de un país (Programa de Estudios e Investigación en Energía, 2003).

Disminuir el consumo individual de energía significa revisar el paradigma de satisfacción personal de los bienes y servicios obtenidos con el uso de energía. Igualmente, la disminución del consumo de energía eléctrica implica demandar menos generación de dicha energía y, por consiguiente, menor uso de recursos naturales energéticos renovables y no renovables, lo que favorece su conservación y el aprovechamiento futuro por parte de las nuevas generaciones. La eficiencia energética significa elegir equipos y tecnologías adecuados para los procesos de conversión con el fin de reducir el consumo. Asimismo, significa utilizar menos recursos energéticos derivados del petróleo importados, conduce a una menor dependencia tecnológica y económica, la cual impide un verdadero desarrollo sostenible con beneficios integrales económicos, sociales y ambientales.

En este artículo centramos nuestra atención en el sector de los ingenios azucareros de Nicaragua. En el país existen cuatro ingenios, de los cuales el San Antonio y el Monte Rosa son agentes del mercado eléctrico por su capacidad de producción de energía y su disponibilidad de potencia eléctrica simultáneamente con la producción de azúcar, rones y biocombustibles. Existen otros dos ingenios que se dedican solamente a la producción de azúcar y energía eléctrica para la autoproducción, que son el ingenio CASUR y el ingenio Montelimar. El objetivo de este artículo es proponer un sistema de gestión integral de la energía que conduzca a su uso eficiente, a través de un proceso de cambio en la forma de su empleo, además de plantear soluciones y medidas de uso eficiente de la energía en los ingenios azucareros, como una manera de contribuir al desarrollo sostenible del país.

\section{Metodología}

Se parte de la descripción de la situación problemática referente a la ineficiencia del empleo de la energía eléctrica y se abordó el rendimiento en su producción en los ingenios. Luego, se propone un proceso de gestión integral de la energía que implica la educación para generar cambios en los valores y actitudes que permitan el ahorro. También, se proponen tecnologías alternativas que ahorran energía eléctrica y el uso de fuentes renovables de energía, para evitar la contaminación ambiental y la emisión de gases de efecto invernadero que son intrínsecas a la generación de energía eléctrica usando combustibles fósiles.

\section{Situación problemática}

Con relación a la gestión energética, Nicaragua presenta un problema de alto índice de intensidad energética que afecta la eficiencia en la producción de bienes y servicios. La intensidad energética es la relación entre el consumo de energía, por ejemplo, en toneladas equivalentes de petróleo (TEP) e indicadores de actividad económica como el Producto Interno Bruto (PIB) (Programa de Estudios e Investigación en Energía, 2003). La intensidad energética mide cuánta energía se consume para producir una unidad de actividad económica. En este sentido, la intensidad energética alcanzó los 0,06 TEP/Millón C\$ PIB de 1994 (Ministerio de Energía y Minas, 20I0). En efecto, la intensidad energética de Nicaragua resulta alta al compararla con el promedio de las economías en desarrollo de ingresos bajos, que en promedio presentan un índice de intensidad energética de 0,022 TEP / Millón \$ PIB (Organización 
de las Naciones Unidas para el Desarrollo Industrial $20 \mathrm{II}$ ). Por tanto, se está usando demasiada energía para la producción, reduciendo la competitividad en los sectores productivos del país.

En cuanto al rendimiento en la producción de energía eléctrica en los ingenios, durante el periodo comprendido entre los años 2000-20 I I, el ingenio San Antonio tuvo un rendimiento promedio de 342,38 Kw-hr/Ton de bagazo, en tanto que la eficiencia promedio del ingenio Monte Rosa fue de 348,39 $\mathrm{Kw}$-hr/Ton de bagazo (Instituto Nicaragüense de la Energía, 2012). Por lo que la eficiencia de generación de ambos ingenios es casi similar, considerando solamente la cantidad de bagazo empleado para generar energía eléctrica en el sistema de cogeneración, de manera separada del resto de productos que se obtienen del procesamiento de la caña de azúcar. No obstante, en el caso de la eficiencia, considerando el empleo integral bruto de la caña de azúcar, en la última zafra de 20 I I-20 I2, la relación entre los kw-hr generados por tonelada métrica de producción de caña para los ingenios es de 93,09 para Monte Rosa, 76,95 para San Antonio, 23,77 para el CASUR y 21,73 para Montelimar (Comisión Nacional de Productores de Azúcar de Nicaragua, 2012). Esto indica que, desde el punto de vista del rendimiento en el sistema de cogeneración, el ingenio Monte Rosa es el más eficiente.

Moreira (2006) indica que con turbinas de vapor es posible generar $100 \mathrm{kw}$-hr/Ton de caña, mientas que Saura y Otero (2008) señalan que a través de la cogeneración se pueden entregar 40 kw-hrl Ton de caña procesada; por su parte, Evans-Pughe (2007) expresa que los países mayores productores de azúcar, como Brasil e India, obtienen una producción de 90 a $140 \mathrm{kw} \mathrm{hr}$ por tonelada de caña. Por lo consiguiente, los ingenios San Antonio y Monte Rosa presentan una intensidad energética muy similar y sus índices de generación de energía eléctrica por cantidad de insumo (tonelada métrica de caña) están dentro de lo que produce la industria de la caña de azúcar a nivel mundial. Los otros dos ingenios nicaragüenses presentan rendimientos de producción energética menores a la industria a nivel mundial. No obstante, se podría mejorar la eficiencia energética de los ingenios optimizando la gestión energética.
Cabe señalar que la energía eléctrica que se produce y consume en Nicaragua proviene de una matriz energética en la cual las energías renovables, incluyendo la biomasa, representan un porcentaje pequeño en relación a las plantas térmicas. Ciertamente, las plantas térmicas que funcionan en base a derivados del petróleo representan el 60\% de la capacidad instalada, las hidroeléctricas el I1\%, las geotérmicas el 4,1\%, las eólicas el 7,12\%, las de biomasa de los ingenios azucareros el I 1,79\% y los sistemas aislados el 1,2\% (Instituto Nicaragüense de Energía, 2012). En el periodo comprendido entre los años 2007 y 2010 se instalaron 243,6 MW de potencia eléctrica en plantas térmicas que funcionan con derivados del petróleo, lo que significó un aumento total del $22,7 \%$ de capacidad instalada con respecto a la capacidad total de potencia del sistema de I 072,59 MW. En este mismo periodo se instalaron solamente $63 \mathrm{MW}$ de plantas eólicas, que representan un 5,8\% del total, y se instalaron 2,597 MW de pequeñas centrales hidroeléctricas, que representan tan solo un 0,24 \% del total de capacidad del sistema (Instituto Nicaragüense de Energía, 20 II). La situación se torna aún más negativa por el hecho de que en el proceso de generación de energía eléctrica se utilizan principalmente sistemas térmicos de generación a base de combustibles fósiles, los cuales degradan el medio ambiente con emisiones de calor y gases de efecto invernadero producto de la combustión del petróleo (Comisión Nacional para el Uso Eficiente de Energía de México, 2009). Por lo tanto, se observa que se están utilizando primordialmente recursos energéticos derivados del petróleo para la generación de energía eléctrica, a pesar del daño que se causa al medio ambiente.

Al generarse la energía eléctrica en las centrales de generación térmica, se producen impactos ambientales que provienen del proceso de combustión de los derivados del petróleo. Todos los contaminantes emitidos por las centrales de generación (a excepción del $\mathrm{CO}_{2}$ ) caen a la superficie de la tierra y su área de dispersión aumenta en función de la altura de la chimenea, la velocidad de salida del gas y la intensidad del viento. Sirva de ilustración el caso del aire que recibe la mayor parte de la contaminación directa en forma de emisiones de polvo y gases contaminantes. De manera que, dependiendo del combustible utilizado para la generación de energía en la central (clase, composición y poder calorífico) 
y de la técnica de combustión, los gases de escape pueden llevar diferentes cantidades de sustancias contaminantes, tales como óxido de nitrógeno (NOx), bióxido de azufre $\left(\mathrm{SO}_{2}\right)$, monóxido de carbono (CO), dióxido de carbono $\left(\mathrm{CO}_{2}\right)$, hidrocarburos no quemados $(\mathrm{HC})$, partículas suspendidas polvo, metales pesados y compuestos orgánicos (Laguna, 2007). De igual manera, el polvo emitido, la mayor parte de los gases contaminantes y productos de transformación atmosférica como el $\mathrm{NO}_{2}$ y nitratos procedentes de las emisiones retornan a la tierra a través de precipitaciones y deposición seca, constituyendo una carga contaminante para el agua y el suelo con potencial de dañar la vegetación y la fauna (Estrucplan, 2012).

Además, el uso de combustibles fósiles como recurso energético en la generación de energía eléctrica ha provocado, por efecto de las condiciones de mercado, que se presente una tendencia al alza incontrolable de los precios del petróleo, causando un aumento en el precio de la energía eléctrica e incidiendo negativamente en la economía nacional. Por ejemplo, en el mercado mayorista de electricidad, el precio se incrementó en promedio entre 2007 y 2010 un 15\% anual (Instituto Nicaragüense de Energía, 201 I).

\section{Cambio del paradigma actual de consumo de energía}

A pesar de los altos costos económicos de la generación de energía eléctrica, los cuales se trasladan directamente al consumidor final, el consumo de esta no disminuye. El consumo de energía eléctrica desde 2000 hasta 2010 aumentó en promedio $4,34 \%$ anual en el sector industrial, $5,14 \%$ en el sector residencial y 3,7\% en el sector comercial (Banco Central de Nicaragua, 20II). Entonces, debemos analizar las razones por las cuales aumenta el consumo de energía, a pesar de que su precio es cada vez mayor.

Una de las causas de la mayor demanda de energía eléctrica es el paradigma del alto consumo energético como signo de alto nivel de vida y de desarrollo sostenido. Este paradigma se debe asociar al hecho de que al sector energético y petrolero internacional le interesa no solo el consumo de sus derivados sino crear dependencia económica y tecnológica, para asegurarse un mercado cautivo. Cabe aquí contrastar dicho paradigma con el concepto de sostenibilidad en el uso de los recursos naturales, que supuestamente posee un origen apolítico pero que al final se ve afectado por los conflictos de poder y de control entre los grupos o sectores sociales por los recursos energéticos; así que no se pueden separar los mecanismos de depredación de la naturaleza de los mecanismos de la explotación económico-social debido a intereses particulares de ciertos grupos de la sociedad (Barkin, 1998). Por lo tanto, es necesario cambiar la cultura actual de consumo energético por una de ahorro y uso racional del recurso.

No obstante, primero se debe analizar si es posible que los individuos cambien sus valores. ReyesSánchez (2012) se refiere a los valores como principios que deseamos que todas las personas desarrollen para beneficio de la humanidad y que no son innatos, se construyen y sí pueden ser modificados. Caduto (1985) señala que los valores como principios, pautas de comportamiento y decisión de actuar, son atributos de carácter individual pero son compartidos por el colectivo mediante la interacción social. En este mismo sentido, Elzo (2004) describe que nuestra sociedad actual está basada en un paradigma de individualismo, donde las soluciones a los problemas sociales deben ser aporte del trabajo de los demás; por lo tanto se deben fomentar valores como la racionalidad, la tolerancia y la solidaridad. Nuévalos (2005) afirma que los valores, sobre todo ambientales, facilitan a los individuos despojarse de intereses particulares y que la conciencia humana social debe sustentar la búsqueda de soluciones que consideren los intereses de todos los habitantes del planeta, sin olvidar a las futuras generaciones. Doffo (2004), por su parte, expresa que los valores ambientales forman a las personas, fomentando actitudes y comportamientos que promueven la alineación como sujetos de interacción en la sociedad y en el medio ambiente. En consecuencia, a través de la formación de valores ambientales adecuados se podrá lograr una participación colectiva en la responsabilidad de la prevención y solución del desperdicio de energía.

De modo que para la formación de valores se puede recurrir a la educación ambiental. Por ejemplo, Foladori y González (200 I) señalan que la educación ambiental permite transmitir al educando un conocimiento que le ayudará a mejorar su relación 
con el medio físico natural y sociocultural. De igual manera, López y Rodríguez (2002) sostienen que la educación ambiental contribuye a la cultura de sostenibilidad a partir de un compromiso social de parte de todos los integrantes de la comunidad, que deben actuar responsablemente en el consumo, la utilización de energía y la generación de desechos. Por lo tanto, se puede inferir que la educación ambiental proporciona la base conceptual de la formación de valores necesarios para facilitar el desarrollo de las aptitudes y habilidades indispensables para la conservación del medio ambiente de manera sostenible y utilizando menos energía eléctrica.

Actualmente se asume que existe una relación entre crecimiento económico y la forma de consumo de energía de una nación; además, que en la forma de consumo de energía inciden las características de la población, tales como longevidad, escolaridad, sectores de consumo, medio, clima, historia, valores, tipo de mercado económico y tipo de sistema político (Sánchez y Álvarez, 2005). Más aún, se conoce que el estilo de vida incide en el consumo de energía eléctrica. Por su parte, Loundon y Dellabitta (1995) afirman que el estilo de vida es una meta que los individuos se imponen para sí mismos, junto con los medios para alcanzarla. Corraliza y Martin (2000) señalan que el estilo de vida es la forma personal en que el ser humano organiza su vida cotidiana y trae consigo características sociales homogéneas como el consumo de energía eléctrica; además, que el estilo de vida lleva inmerso un conjunto de valores, normas, conductas y hábitos de uso, relacionados con la cultura y la expresión individual y social. Asimismo, Novo (2006) llama a evitar los estilos de vida basados en la velocidad y el consumismo con rapidez, que son agresivos con el medio ambiente. Por lo consiguiente, los cambios en la conducta ambiental, como el ahorro en el consumo de energía eléctrica, están relacionados con transformaciones de valores y aspiraciones que orientan la vida de un individuo, sus características y las de la comunidad en que habita.

En consecuencia, se hace necesario impulsar cambios en los diferentes sectores económicos y sociales; de tal manera que el nuevo paradigma tendría que llevarnos a detener selectivamente el crecimiento material en sociedades desarrolladas, lo que no significa detener el desarrollo humano, ni implicaría detener la producción de bienes y servicios (Riechmann \& Naredo, 1995). Al contrario, el cambio significa consumir recursos energéticos considerando la sostenibilidad a largo plazo y promoviendo valores que fomenten niveles de consumo que permanezcan dentro de los límites de lo ecológicamente posible.

Por tanto, para romper el modelo actual es necesario un cambio de cultura energética, que implica romper un paradigma que dicta que un alto consumo energético es sinónimo de desarrollo y mejor estilo de vida. Actualmente, los patrones de consumo de los países más ricos están relacionados con un sistema productivo que solo prospera generando nuevas demandas de bienes para continuar creciendo (Barkin, 1998). El consumismo ha hecho que las empresas se enfoquen en producir a gran escala, olvidándose de prácticas de producción eficientes.

\section{Propuesta de gestión y eficiencia energética}

En las empresas industriales, se debe considerar un cambio de paradigma de consumo y gestionar con eficiencia la energía, para así potenciar el impacto positivo que el uso racional de esta tiene sobre el medio ambiente (Campos et al., 2008). Está claro que una gestión eficiente de la energía mejora la productividad y competitividad empresarial. Es por ello que lograr el uso racional y eficiente de la energía en los ingenios azucareros de Nicaragua significa hacer cambios en su sistema de cultura y gestión organizacional, generando productividad y equilibrio ambiental.

Para alcanzar este objetivo se propone implementar un modelo de Gestión Integral de la Energía (GIE), que es un conjunto estructurado de procedimientos y actividades diseñados para integrarse al sistema de gestión organizacional de la empresa industrial. Este modelo contempla un conjunto de medidas, normas, procedimientos, valores y actuaciones que permitan la materialización de los objetivos de eficiencia energética. El sistema está compuesto por tres elementos fundamentales: la decisión estratégica, la instalación y la operación del sistema (Campos et al., 2008).

Según Vega y Álvarez (2005), en este sistema, la decisión estratégica comienza con la alineación de la gerencia de la empresa con el objetivo de ahorrar energía. Además, todo el personal debe comprender la importancia de este ahorro como medio para 
mejorar la rentabilidad de la empresa y contribuir con el entorno ambiental. Es decir, se adquiere el compromiso de involucrarse en todas las acciones del proceso de gestión del ahorro de energía. Por otra parte, la instalación del proceso de gestión de energía implica la sensibilización y capacitación del personal de la empresa, de forma que la meta será el cambio de paradigma de consumo, haciéndose necesaria una educación que puede retomar los elementos metodológicos de la educación ambiental. De esta forma, se propone impulsar la educación para la eficiencia energética que buscará crear valores culturales para evitar el deterioro ambiental y utilizar los conocimientos científico-tecnológicos de manera eficiente para su conservación.

Otra parte de la gestión integral de la energía consiste en realizar un diagnóstico de la empresa, que no es más que una revisión de cuánto y cómo se consume la energía. Se deben buscar posibles fugas o desperdicios para eliminarlos, así como establecer estándares de consumo que sirven de referencia para el control del gasto energético. También se debe establecer un plan de medidas de ahorro con metas y recursos definidos que incluya: acomodos de carga, es decir, evitar en lo posible que todos los receptores eléctricos consuman energía al mismo tiempo, reingeniería de los procesos industriales para optimizar los procesos y medidas de educación, sensibilización e información para detectar consumos innecesarios de energía eléctrica. Asimismo, la gestión energética incluye el empleo de tecnologías de alta eficiencia en iluminación y en conversión de energía, así como sistemas de climatización y refrigeración eficientes.

En cuanto a la operación del sistema, esta incluye el monitoreo de las actividades del plan de ahorro energético, su seguimiento y evaluación de las buenas prácticas de operación e implementación de proyectos de tecnología de alta eficiencia. Por último, se deben hacer evaluaciones periódicas de los planes y proporcionar reconocimientos a las personas que destaquen en la implementación de las medidas de ahorro, porque son ellas las que harán el cambio del paradigma de consumo y para lograr un compromiso y cambio de actitud. Tanto en la formulación del plan de ahorro como en el desarrollo de todas las medidas que se apliquen se debe involucrar a todo el talento humano de las empresas.

\section{Propuesta de implementación de tecnologías alternativas y fuentes renovables de energía}

Como parte de la propuesta para el cambio del consumo de energía en los ingenios azucareros de Nicaragua, se plantea la producción más limpia como estrategia que reduce costos y genera ganancias reduciendo el desperdicio (Programa de las Naciones Unidad para el Ambiente, 2004). Producción más limpia significa vigilar el proceso productivo de una empresa, comparar las entradas de recursos con sus salidas y eliminar los residuos e ineficiencias. Es así que se plantea la desmaterialización, como enfoque paradigmático alternativo. Esta consiste en lograr un incremento de la productividad, mediante la reducción del uso de los insumos utilizados para la producción; contribuyendo a la ecoeficiencia. Es decir, producir más con menos, utilizando menos recursos ambientales y menos energía en el proceso productivo, reduciendo desechos y atenuando la contaminación (Elizalde, 2009).

Por otra parte, Téllez y Ávila (2009) indican que es posible integrar las energías alternativas como parte de opciones que favorecen el uso sustentable de la energía, de tal manera que se pueda aprovechar la energía solar pasiva, denominada también arquitectura solar pasiva o bioclimática, ahorrando energía hasta en un 70\% durante el tiempo de utilización, obteniéndose a la vez un óptimo confort térmico. Según estos autores, la arquitectura bioclimática consiste en analizar las condiciones climáticas, el entorno de los edificios y las cargas internas (número de ocupantes y dispositivos de acondicionamiento auxiliar), con el fin de elaborar un balance energético que permita aprovechar el entorno para mejorar la climatización. Asimismo, el balance energético es un procedimiento en el cual se analizan las ganancias, pérdidas y acumulación de energía en las edificaciones para evitar la ganancia de calor desde el exterior; como resultado se obtienen recomendaciones como el empleo de protecciones en forma de aleros, toldos o persianas o bien conectar el edificio con sumideros de calor, entre otras. Además, se pueden usar elementos como tejados, ventanas y proponer nuevos diseños arquitectónicos o reacondicionar la infraestructura existente con el objetivo de conseguir el mínimo 
consumo de energía, el máximo confort térmico, de iluminación y las condiciones necesarias para el buen desempeño de labores en las empresas industriales.

Otra forma de aplicación de la arquitectura bioclimática es propiciar la iluminación natural diurna de oficinas y naves industriales. La iluminación natural se consigue mediante elementos translúcidos y reflectantes en el interior de los edificios; la luz difusa se aprovecha con pinturas blancas o claras, la finalidad es permitir la entrada de luz natural y reducir la necesidad de luz artificial. Igualmente, para aprovechar al máximo la luz natural se puede coordinar la apertura de espacios en paredes con el régimen horario de uso de iluminación en los locales (Monroy, 2006). Además, se pueden emplear focos solares que permitan aprovechar la luz solar temprano en la mañana y al atardecer (Natural Ligth Energy Systems, 20 I2).

Otra propuesta para el ahorro energético es la llamada domótica, que es una combinación de tecnología electrónica e informática, que integra el control y supervisión de los elementos existentes en un local de manera autónoma. La domótica se concentra en cumplir con el propósito de suministrar energía eficientemente, brindar comodidad, seguridad y comunicación en los procesos, utilizando los últimos avances técnicos de la electrónica y la informática. La domótica permite: a) el ahorro de energía al optimizar recursos automatizando los procesos, b) el ahorro de tiempo de producción controlando cada etapa de acuerdo a una programación óptima, c) el incremento de los niveles de seguridad e higiene laboral al monitorear condiciones de confort, y d) brindar información necesaria oportuna de vigilancia (Rodríguez et al., 200 I). Así, el uso de la domótica permite gestionar y monitorear el uso de la energía en los sistemas de iluminación, climatización y diferentes equipos, ahorrando energía eléctrica.

Por otra parte, la energía solar térmica también se puede emplear en el sector industrial de los ingenios azucareros como sustituto de combustibles fósiles en la producción de calor. Esta tecnología emplea sistemas de colectores solares en los que un fluido, generalmente un líquido, toma el calor de los rayos solares y los transmite a otro fluido y lo calienta mediante captadores solares planos. La conversión térmica de baja temperatura permite la obtención de agua caliente hasta $80^{\circ} \mathrm{C}$ para diversos usos: agua caliente sanitaria, agua para procesos industriales, refrigeración ambiental e instalaciones de secado (Téllez y Ávila, 2009).

Asimismo, la propuesta busca la implementación de la cogeneración en todos los ingenios azucareros. Según Blarke y Dotzauer (20 I I), la cogeneración es la producción combinada y secuencial de calor y electricidad desde una fuente común de energía. Por su parte, Moon et al. (20 I I) expresan que las industrias con sistemas energéticos de cogeneración, que producen electricidad a través de turbinas de vapor, tienen eficiencias de conversión de energía de entre $10 \%$ y $35 \%$ en función del tamaño y el fabricante de los equipos. Además, los sistemas de cogeneración presentan una eficiencia global mayor al $80 \%$ para plantas de gran capacidad (Moon et al., 20 I I, Siler et al., 20।2).

\section{Conclusiones}

La gestión eficiente de la energía y el cambio de la cultura de consumo contribuirán a superar el problema de la alta intensidad energética que presenta Nicaragua y en especial el sector industrial de los ingenios azucareros. Este cambio de cultura implica utilizar menos energía eléctrica y cambiar nuestra forma de consumirla, o sea, usarla de manera eficiente. También significa revisar el paradigma de satisfacción personal de los bienes y servicios obtenidos con el uso de energía.

En Nicaragua existe dependencia de recursos energéticos no renovables importados, derivados del petróleo, los cuales se usan para generar la energía eléctrica; por lo que la disminución de su consumo implicaría demandar menos generación y, por consiguiente, menor uso de recursos energéticos y de inversiones en la infraestructura asociada al transporte y suministro de energía. Por lo anterior, se hace necesario un nuevo paradigma que contradiga la creencia de que un alto consumo de energía es señal de alto nivel de vida y de desarrollo. Por el contrario, se insta a consumir menos recursos energéticos, considerando la sostenibilidad a largo plazo y dentro de los límites de lo ecológicamente posible.

Por otro lado, se puede afirmar que es posible hacer un cambio del paradigma de consumo energético en el sector industrial de los ingenios azucareros 
de Nicaragua. Para ello se propone un nuevo paradigma mediante el sistema de gestión integral de la energía. Además, como alternativa para disminuir el uso de combustibles fósiles, se plantea la introducción de innovaciones tecnológicas de energías renovables, especialmente el empleo de biomasa en sistemas de cogeneración, la energía solar pasiva o bioclimática y la domótica.

\section{Bibliografía}

Banco Central de Nicaragua (BCN) (201 I). Estadísticas económicas anuales: principales indicadores macroeconómicos. Obtenido el 8 de junio de 2012 desde http://www.bcn.gob. ni/estadisticas/economicas_anuales/index.html?\&val=2

Banco Central de Nicaragua (BCN) (20II). Nicaragua en cifras. Obtenido el II de junio de 2012 desde http://www. bcn.gob.ni/estadisticas/economicas_anuales/nicaragua_en_ cifras/20 I I/Nicaragua_cifras_20 I I.pdf

Barkin, David. (1998). Riqueza, pobreza y desarrollo sustentable. México: Editorial Jus y Centro de Ecología y Desarrollo.

Blarke, M. \& Dotzauer, E. (20II). Intermittency-friendly and high-efficiency cogeneration: Operation of cogeneration with compression heat pump, fuel gas, heat recovery, and intermediate cold storage. Energy 36, 6867-6878. Obtenido el II de Junio de 2012 desde http://login.oaresciences.org

Campos, J., Prias, O., Ospino, I., Quispe, E., Vidal, J. \& Lora, E. (2008). Modelo de gestión energética para el sector productivo nacional. El hombre y la máquina, 30, |8-31. Obtenido el II de junio de 2012 desde http://ingenieria.uao.edu.co/hombreymaquina/revistas/30\%202008-I/ Articulo\%202\%20H\&M-30.pdf

Caduto, M.J. (1985). A Guide on EnvironmentalValues Education. Environmental Education Series 13, 7-28. International Environmental Education Programme. Division of Science, Technical and Environmental Education, UNESCO.

Comisión Nacional de Productores de Azúcar de Nicaragua (CNPA). (20/2). Datos de producción de azúcar de los ingenios azucareros de Nicaragua. Obtenido el 19 de julio de 2012 desde http://www.cnpa.com.ni/index. php?option=com_content\&view=article\&id=27\&ltem id $=30$

Comisión Nacional para el Uso Eficiente de Energía de México (CONNUE) (2009). Metodologías para la cuantificación de gases de efecto invernadero y consumos energéticos evitados por el aprovechamiento sustentable de la energía. Obtenido el I I de junio de 2012 desde http://www.conuee. gob.mx/work/files/metod_gei_cons_evit.pdf

Corraliza, J., Martin, R. (2000). Estilos de vida, actitud y comportamiento ambiental. Medio ambiente y comportamiento humano I ( I): 35-56.

Doffo, D. (2004). Educación ambiental como educación en valores. Ponencia presentada en el VI Encuentro Corredor de las Ideas "Sociedad Civil, Democracia e integración". Montevideo.

Elizalde, A. (2009). ¿Qué desarrollo puede llamarse sostenible en el siglo XXI? La cuestión de los límites y las necesidades humanas. Revista de educación, número extraordinario, 53-75.

Elzo, J. (2004). La educación del futuro y los valores. Ponencia impartida dentro del ciclo Debates de educación. Fundación Jaume Bofill y Universitat Oberta de Catalunya. Barcelona. Obtenido el II de junio de 2012 desde http://www. solache.com/laeducaciondelfuturoylosvalores.pdf.

Estrucplan (2012). Impactos ambientales de las centrales térmicas. Argentina. Obtenido el II de junio de 2012 desde http://www.estrucplan.com.ar/producciones/entrega. asp?identrega $=297$

Evans-Pughe, C. (2007). Sugar Rush. Engineering \& Technology 2(2), 38-42. Obtenido el 27 de julio de 2012 desde http:// web.ebscohost.com

Foladori, G. \& González, E. (200I). En pos de la historia de la educación ambiental. Tópicos en Educación Ambiental, 3(8): 28-43. Obtenido el 7 de junio de 2012 desde http://anea. org.mx/Topicos/T\%208/Paginas\%2028\%20-\%2043.pdf

Instituto Nicaragüense de Energía (INE) (20I I). Estadísticas de capacidad instalada. Obtenido el 16 de agosto de 2012 desde http://www.ine.gob.ni/DGE/estadisticas/serieHistorica/Capacidad_Instalada.pdf

Instituto Nicaragüense de Energía (INE) (20/2). Rendimiento por tipo de planta. Obtenido el 16 de agosto de 2012 desde http://www.ine.gob.ni/DGE/estadisticas/serieHistorica/Rendimientos_9l - I I.pdf

Instituto Nicaragüense de Energía (INE) (20I2). Capacidad por tipo de planta. Obtenido el 16 de agosto de 2012 desde http://www.ine.gob.ni/DGE/estadisticas/serieHistorica/ Capacidad_Instalada_9 |- II.pdf

Laguna, I. (2007). Generación de energía eléctrica y medio ambiente. Obtenido el II de junio de 2012 desde http:// www2.ine.gob.mx/publicaciones/gacetas/367/energiamed. html\#top

López, E. \& Rodríguez, A. (2002). Programa Jalpa, Limpio y Verde. Políticas públicas y Educación Ambiental para comunidades saludables. Horizonte sanitario 3, 26-37.

Loundon, D. \& Dellabita, A. (1995). Comportamiento del consumidor, conceptos y aplicaciones. México: McGraw Hill.

Ministerio de Energía y Minas de Nicaragua (20l0). Balance energético nacional 2010. Obtenido el II de junio de 2012 desde http://www.mem.gob.ni

Ministerio de Energía y Minas de Nicaragua (20l0). Guía del Inversionistas. Obtenido el II de junio de 2012 desde http:// www.mem.gob.ni/media/file/ELECTRICIDAD\%20Y\%20 RECURSOS/R-RENOVABLES\%20C-ENERGIA/Guia\%20 del\%2OInversionista\%20Sector\%20Electrico\%20de\%20 Ncaragua\%2020 10\%20\%2009Sept20 I0.pdf

Monroy, M. (2006). Calidad ambiental en la edificación para Las Palmas de Gran Canaria: Guía de aplicación de diseño de ilumi- 
nación, aire y calor. Manual de Diseño ICARO. Departamento de Construcción Arquitectónica, Servicio del medio ambiente del Ayuntamiento de la Palmas de Gran Canaria.

Moon, J., Woon, L. \& Uen, L. (20II). Economic analysis of biomass power generation schemes under renewable energy initiative with Renewable Portfolio Standards (RPS) in Korea. Bioresource Technology 102(20):9550-9557. Obtenido el II de junio de 2012 desde http://login.oaresciences. org/whalecomwww.sciencedirect.com/whalecom0/science/ article/pii/S09608524I I 009916

Moreira, J. (2006). Global biomass energy potencial. Mitigation and Adaptation Strategies for Global Change II, 313-342. Obtenido el 30 de agosto de 2012 desde http://www. springerlink.com/content/c77g5 I 5623 | 5 | 47v/fulltext.pdf

Natural Light Energy Systems (2012). Commercial Tubular Skylights. Obtenido el II de junio de 2012 desde http:// www.nltubular.com/index.html

Novo, M. (2006). El desarrollo sostenible. Madrid: Pearson Prentice Hall, S.A.

Nuévalos, C. 2005. La educación en valores ambientales. Ethos educativos, 32, 83-105. Obtenido el II de junio de 2012 desde http://www.imced.edu.mx/Ethos/Archivo/32/32-83. pdf

Organización de las Naciones Unidas para el Desarrollo Industrial (2011). Informe sobre el Desarrollo Industrial: Eficiencia energética industrial para la creación sostenible de riqueza. Obtenido el 19 de julio de 2012 desde http://www. unido.org/fileadmin/user_media/Publications/IDR/20 I I/ IDR_Overview_Spanish.PDF

Organización Latinoamericana de la Energía (OLADE) (2009). Informe de estadísticas energéticas. Obtenido el II de junio de 2012 desde http://www.olade.org.ec

Programa de Estudios e Investigaciones en Energía (2003). Estudio de las relaciones entre la eficiencia energética y el desarrollo económico. Sociedad Alemana para la Cooperación. Obtenido el I I de junio de 2012 desde www.prien.cl/documentos/GTZ_Eficiencia\%20y\%20desarrollo.pdf

Programa de las Naciones Unidad para el Ambiente (2004). Guidelines for integration of cleaner production and energy efficiency. US: United Nations Publication.
Ramírez, R. (2008). Sostenibilidad y Eficiencia Energética en la Industria. CITCEA. Obtenido el II de junio de 2012. Disponible en http://es.scribd.com/doc/23525965/EficienciaEnergetica-en-la-Industria

Reyes-Sánchez, L. (20/2). Los valores. Universidad Nacional Autónoma de México.

Riechmann, J. \& Naredo, J. (1995). Desarrollo sostenible: la lucha por la interpretación. Obtenido el II de junio de 2012 desde http://www.ambiente.gov.ar/infotecaea/descargas/ riechman0l.pdf

Rodríguez, W., Pinzón, Z. \& Naranjo, P. (200 I). Domotica, ciencia ficción hecha realidad. Ciencia e Ingeniería Neogradina II, 59-65. Obtenido el II de junio de 2012 desde http://redalyc.uaemex.mx/redalyc/src/inicio/ArtPdfRed. jsp?iCve=91 101110 .

Saura, G. \& Otero, M. (2008). Posibilidades y alternativas de la diversificación del sector azucarero. ICIDCA 17. Obtenido el 23 de julio de 2012 desde http://redalyc.uaemex.mx/ redalyc/pdf/223 I/223 I 206670 I I.pdf

Sánchez, J. \& Álvarez, P. (2005). Planificación energética y desarrollo sostenible. Obtenido el 6 de junio de 2012 desde http://eltroldesdesucaverna.com/homearch. php?nta=37\&men=m I \&smen=Tesis\%20Doctorado

Siler, E., Granger, M. \& Lima, I. (20।2). Distributed cogeneration for commercial buildings: Can we make the economics work?. Energy Policy 42, 580-590. Obtenido el día II de junio de 2012 desde http://login.oaresciences.org

Téllez, M. \& Ávila, A. (2009). Energía Solar Térmica. España: Centro de Educación a Distancia para el Desarrollo Tecnológico y Económico y Centro de Investigaciones Energéticas, Medioambientales y Tecnológicas del Ministerio de Educación y Ciencias.

United Nations Environment Program (2004). Guidelines for integration of cleaner production and energy efficiency. United Nations Publication.

Vega, P. \& Álvarez, P. (2005). Planteamiento de un marco teórico de la Educación Ambiental para un desarrollo sostenible. Revista Electrónica de Enseñanza de las Ciencias 4(I). Obtenido el II de junio de 2012 desde http://www.saum. uvigo.es/reec/volumenes/volumen4/ART4_Vol4_NI.pdf 